\title{
EchoGéo
}

$56 \mid 2021$

Les dynamiques spatiales contemporaines de la Russie

\section{Wikipédia à tous les étages. Usages de l'encyclopédie pour l'enseignement en géographie}

\section{Hadrien Commenges}

\section{(2) OpenEdition}

\section{Journals}

Édition électronique

URL : https://journals.openedition.org/echogeo/21453

DOI : $10.4000 /$ echogeo. 21453

ISSN : 1963-1197

Éditeur

Pôle de recherche pour l'organisation et la diffusion de l'information géographique (CNRS UMR 8586)

Référence électronique

Hadrien Commenges, « Wikipédia à tous les étages. Usages de l'encyclopédie pour l'enseignement en géographie », EchoGéo [En ligne], 56 | 2021, mis en ligne le 25 juin 2021, consulté le 31 juillet 2021. URL : http://journals.openedition.org/echogeo/21453 ; DOI : https://doi.org/10.4000/echogeo.21453

Ce document a été généré automatiquement le 31 juillet 2021.

EchoGéo est mis à disposition selon les termes de la licence Creative Commons Attribution - Pas d'Utilisation Commerciale - Pas de Modification 4.0 International (CC BY-NC-ND) 


\title{
Wikipédia à tous les étages. Usages de l'encyclopédie pour l'enseignement en géographie
}

\author{
Hadrien Commenges
}

\section{Introduction}

1 D'après Wikipédia, "Wikipédia est une encyclopédie universelle, collaborative et multilingue, créée par Jimmy Wales et Larry Sanger le 15 janvier 2001 » (Source Wikipédia, entrée "Wikipédia"). L'encyclopédie compte aujourd'hui (1 $1^{\text {er }}$ février 2020) 395 millions de pages pour l'ensemble des wikis (Wikipédia, Wikibooks, Wikinews, Wikiquote, etc.) et l'ensemble des langues. L'édition anglaise de Wikipédia compte 6 millions de pages, les autres langues principales comptent entre 1 et 3 millions de pages : 2,4 millions pour l'Allemand, 2,2 millions pour le Français, 1,6 millions pour l'Italien, pour l'Espagnol et pour le Russe, 1,2 millions pour le Japonais, 1,1 million pour le Chinois ${ }^{1}$.

Depuis sa naissance, Wikipédia a fait l'objet de nombreux travaux dans des disciplines variées (Broudoux, 2015). Dans le domaine de l'informatique, l'univers Wikipédia (Wikidata, DBPedia) a été le terrain d'essai des méthodes d'extraction d'information (IR-information retrieval), de traitement du langage naturel (NLP-Natural Language Processing) et de construction d'ontologies, méthodes à la base du web sémantique (Medelyan et al., 2009; Mehdi et al., 2017). En géographie et en géomatique, Wikipédia peut être considéré comme un espace de constitution d'une information géographique collaborative (VGI - Volunteered Geographic Information), Wikipédia est aussi un corpus et un terrain d'essai pour mettre en place des méthodes d'extraction de l'information géographique (GIR-Geographic information retrieval), un problème classique dans ce domaine étant de désambiguiser des toponymes (Overell et Rüger, 2008), par exemple différencier dans un texte « London » au Royaume-Uni de « London » dans l'Ontario. En sciences politiques (Levrel, 2006 ; Cardon et Levrel, 2009; Weltevrede et Borra, 2016), 
les travaux visent à examiner les traces numériques laissées par la production du contenu de l'encyclopédie, traces révélatrices de l'actualité, des controverses, ou encore de la structuration d'une communauté de contributeurs. Wikipédia est un objet particulièrement riche pour l'analyse et la visualisation des controverses, ce qui a mené au développement de méthodes et d'outils dédiés comme par exemple le logiciel Contropedia ${ }^{2}$. Ce logiciel permet d'entrer dans les controverses révélées par l'encyclopédie à partir de l'analyse de la dynamique des contributions, qu'il s'agisse du conflit israélo-palestinien ou du terme Feta (Pentzold et al., 2017). L'exemple de la feta montre une controverse à la fois sur le nom du fromage, sur son contenu (chèvre, brebis et/ou vache) et surtout sur son origine (Grèce, Macédoine, Bulgarie, Turquie).

3 L'usage de Wikipédia comme objet de recherche n'a jamais posé problème, en revanche, l'usage de Wikipédia pour l'enseignement, en particulier pour l'enseignement universitaire, a toujours posé problème. Une majorité d'enseignants sont méfiants visà-vis de l'encyclopédie et recommandent explicitement aux étudiants de ne pas l'utiliser (Knight et Pryke, 2012 ; Haslam, 2017). Une phrase de Pierre Assouline (2007) a très bien résumé cette méfiance : "Wikipédia est la seule encyclopédie au monde où n'importe qui peut écrire n'importe quoi ». Malgré ces recommandations, les élèves et les étudiants utilisent massivement Wikipédia pour la recherche d'information (Knight et Pryke, 2012; Sahut et al., 2015), tout en considérant, après leurs enseignants, qu'il s'agit d'une source illégitime. Ainsi, l'encyclopédie dispose d'un statut ambivalent, elle est à la fois utilisée et méprisée par les étudiants et les enseignants alors que très peu d'entre eux sont contributeurs. Finalement, tout le monde a un avis sur l'encyclopédie alors que tout ce même monde ignore comment son contenu est produit. Certains auteurs déjà cités (Knight, Pryke, Sahut) ont mené des enquêtes pour en quantifier l'usage et la contribution. Sans avoir mis en place une démarche d'enquête, je questionne régulièrement mes collègues et je questionne chaque année les étudiants du module de L3 dont il sera question par la suite: jamais je n'ai rencontré un contributeur à l'encyclopédie.

4 Cette rareté pose question d'autant que les initiatives sont variées et déjà anciennes. En 2010 a été lancé un programme "Wikipédiens en résidence ", qui consiste à placer un Wikipédien expert dans une institution pour qu'il y développe des usages liés à l'encyclopédie. Ces résidences se font majoritairement dans des institutions culturelles (musées, bibliothèques, fondations, voir la liste complète ${ }^{3}$ ), mais parfois dans des universités (Berkeley, Edimbourg, Haifa, Harvard). En France cette pratique est rare, la seule institution française ayant accueilli un Wikipédien en résidence jusqu'à maintenant est le Palais de Versailles. Wikimedia a lancé dès 2010 un programme destiné à généraliser l'usage de l'encyclopédie dans l'enseignement (Wikipedia Education Program ${ }^{4}$ ). La fondation propose des documents pour encourager cette démarche. La brochure Utiliser Wikipédia avec vos étudiants présente ainsi 14 expériences pédagogiques menées avec Wikipédia (dont aucun exemple français). La fondation propose aussi des outils, en particulier les projets pédagogiques ${ }^{5}$ qui permettent de créer une feuille de route, de planifier des tâches, d'organiser les groupes et de centraliser les contributions effectuées par les étudiants ${ }^{6}$. Certains organismes, comme l'American Sociology Association ont lancé des initiatives visant à encourager l'usage de Wikipédia dans le monde académique (Sociology in Wikipedia Initiative).

5 Wikipédia reste très peu intégrée aux enseignements universitaires alors qu'elle présente des avantages certains. En particulier, l'utilisation de l'encyclopédie dans le 
cadre universitaire est particulièrement pertinente puisque Wikipédia est par définition un lieu qui fait le lien entre la production et la transmission de connaissances. Les arguments en sa faveur ont été développés par plusieurs auteurs, en particulier par Haslam (2017) pour qui la conclusion est sans appel : «Wikipedia can and should have a vital role in humanities higher education, including preparing students to write better and to do scholarly research ${ }^{7}$. Wikipédia a été intégrée aux pratiques d'enseignement dès ses premières années d'existence, mais dix ans plus tard, Knight et Pryke (2012) parlaient toujours d'une minorité d'enseignants utilisant Wikipédia dans leurs enseignements. Vingt ans plus tard (Cummings, 2019), l'usage de Wikipédia s'est répandu mais continue de sembler minoritaire, a fortiori dans l'université française. Malgré tout, les usages dans l'enseignement restent rares et conservent un statut expérimental.

6 L'objectif de ce texte est de prendre position en faveur d'une généralisation de l'usage de Wikipédia pour l'enseignement universitaire, en SHS et en géographie en particulier. Il ne faut pas lire cette prise de position comme celle d'un Wikipédien militant. Il s'agit plutôt de l'évaluation a posteriori de l'intérêt et du succès obtenu en introduisant Wikipédia dans des modules d'enseignement. Il s'agit de trois modules donnés dans l'UFR de géographie de l'Université Paris 1-Panthéon Sorbonne: un module de statistique en L2, un module d'études urbaines en L3 et un module du master Carthagéo, master interuniversitaire de géomatique (Université Paris 1, Université Paris 7, École Nationale des Sciences Géographiques). Ces trois cas d'usage pédagogique sont présentés successivement, ils sont incomparables et d'un intérêt variable selon le profil de qui s'y intéresse. L'intérêt ici est surtout de les juxtaposer pour montrer comment l'usage de Wikipédia peut être introduit sur des thèmes variés et à des niveaux d'enseignement différents.

\section{Wikipédia comme source d'information}

7 Le chapeau Wikimedia abrite plusieurs projets - Wikipédia, Wiktionary, Wikiquote, Wikidata, Wikimedia Commons, etc. - qui peuvent en premier lieu être utilisés comme source d'information mobilisable dans les enseignements. Dans ce cadre il faut distinguer 1) l'utilisation comme source (information extraite de Wikimedia pour ellemême) et 2) l'utilisation comme trace numérique (information sur le dispositif encyclopédique lui-même).

\section{Wikipédia comme contenant}

8 Dans le premier cas, et sans prétendre à l'exaustivité, Wikimedia Commons est une base de 60 millions de fichiers média (en février 2020), image, son et vidéo. Ces fichiers sont librement réutilisables, dans des supports de cours, des articles ou des ouvrages, soit parce qu'ils sont sous licence libre ${ }^{8}$ soit parce qu'ils sont tombés dans le domaine public. Un exemple, parmi 60 millions d'autres, Dehli, great capital of India (1909) ${ }^{9}$ extrait d'une collection de vidéos historiques du British Film Institute tombées dans le domaine public.

9 La question des licences n'est pas anodine pour la production de textes destinés à l'enseignement et la recherche. Par exemple, il n'existe pas sous licence libre de photographie officielle française des présidents français. Les seules photos réutilisables 
de ces présidents sont issues de services d'archive étrangers : archives allemandes pour De Gaulle ${ }^{10}$, archives hollandaises pour Mitterand ${ }^{11}$, archives américaines pour Macron $^{12}$. Les photos officielles des présidents français sont protégées par le copyright $\mathrm{du}$ photographe alors que dans d'autres pays les photos officielles tombent dans le domaine public, comme l'indique la mention des archives américaines: «This file is a work of an employee of the Executive Office of the President of the United States, taken or made as part of that person's official duties. As a work of the U.S. federal government, it is in the public domain $»^{13}$.

Par ailleurs il est possible d'extraire de l'information de Wikimedia de façon plus massive et systématique. Plusieurs approches sont envisageables.

11 1) Utiliser les outils déjà mis en place dans l'encyclopédie, approche facilitée par le nombre et la variété de documents de synthèse, en particulier les listes. Il existe des milliers de listes (nombre variable selon la langue), il existe des listes qui recensent les listes et il existe même une liste des listes des listes ${ }^{14}$. À titre d'exemple, une liste des émeutes ${ }^{15}$, une liste des épidémies ${ }^{16}$, une liste des cours d'eau frontaliers ${ }^{17}$, une liste des langues disparues ordonnée par date d'extinction ${ }^{18}$ dans laquelle on apprend que la dernière langue disparue est le mandan, le 9 décembre 2016. Ces exemples ne sont pas anecdotiques, ce type de recueils est à la fois très difficile à trouver dans d'autres sources d'information et aussi très difficile à reconstituer par un seul individu.

2) Utiliser des outils mis en place autour de Wikipédia pour parcourir, indexer et stocker des informations. Wikipédia est un corpus qui contient à la fois du texte non structuré (paragraphes des articles) et du texte structuré (par exemple chaque pays comporte une « infobox » qui contient la population, la surface, la capitale, l'hymne, etc.). Ce corpus peut être parcouru (web crawling) et moissonné (web scrapping) avec une large gamme d'outils logiciels. Une recherche sur le dépot de code github avec les motsclés wikipedia et crawler renvoie 43 résultats (en février 2020) ${ }^{19}$. Parmi ces programmes, certains sont confidentiels, mal documentés, peu ou pas maintenu. En revanche, certains laboratoires mettent à disposition des outils tout à fait accessibles, en particulier Digital Methods Initiative à l'université d'Amsterdam ${ }^{20}$ et le Médialab de Sciences $\mathrm{Po}^{21}$.

13 3) Développer des programmes pour requêter et pour moissonner des informations dans Wikipédia et les sites associés. Il existe des bibliothèques de fonctions dédiées dans plusieurs langages informatiques, en particulier $\mathrm{R}$ (WikipediR, WikipediaR, wikipediatrend) et Python (wikipedia) Ce point sera développé dans la dernière section de cet article, puisqu'il peut s'agit d'un objectif d'apprentissage pour les formations en géomatique.

\section{Wikipédia comme espace de production}

Wikipédia n'est pas seulement un dispositif de diffusion de contenus, c'est aussi un espace de production (Levrel, 2006). S'il est possible de constituer des corpus à partir des informations contenues dans l'encyclopédie, il est aussi intéressant d'examiner les traces numériques laissées par la production de ce contenu. Ces traces peuvent être envisagées de différentes façons, au moins deux directions doivent être mentionnées : traces révélatrices de l'actualité et traces révélatrices des pratiques de la communauté des contributeurs. 
Plusieurs travaux ont examiné les logiques spatiales et temporelles liées à la production de l'actualité à partir de corpus de médias. Les volumes 2016/1 et 2016/2 de la revue L'Espace géographique en donnent un aperçu (Beauguitte et al., 2016). Wikipédia n'est pas conçue pour ce genre d'usages mais il se trouve que depuis sa naissance l'encyclopédie a été utilisée comme dispositif de journalisme participatif (Keegan et al., 2013; Doutreix, 2020). On peut donc utiliser les traces de la production des contenus comme s'il s'agissait d'un corpus médiatique. La série temporelle des contributions à une page est un sismographe de l'actualité. (illustration 1). Des analyses plus fines des dynamiques de contribution permettent de travailler sur l'actualité des controverses (Pentzold et al., 2017).

Illustration 1 - Wikipédia comme sismographe, l'exemple de Mandela

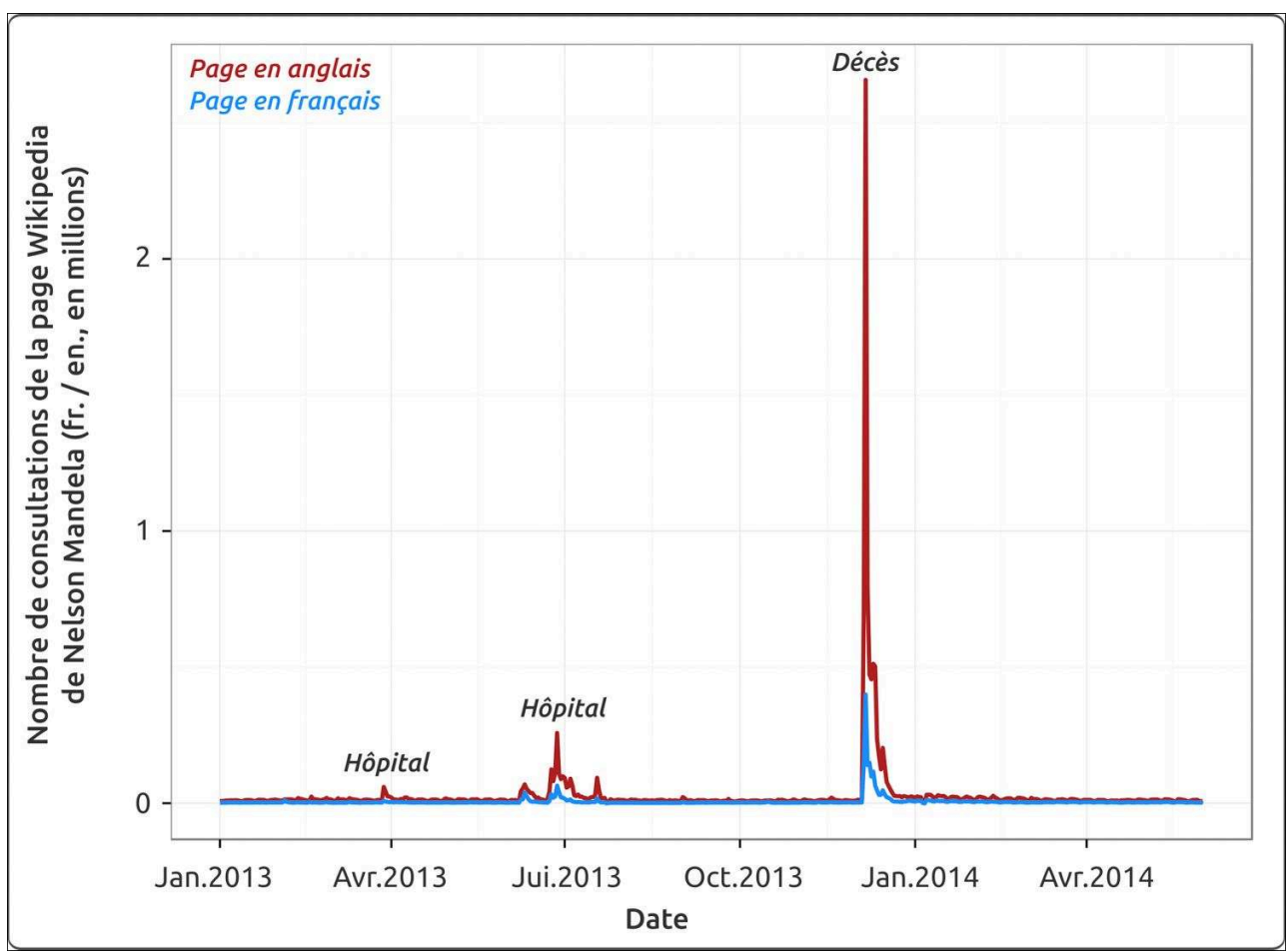

À partir de données moissonnées sur Wikipédia, j'ai construit un support de TD destiné à l'introduction du cours de statistique en L2. Pour chaque pays du monde et pour chacune des 14 langues les plus parlées au monde ${ }^{22}$ on extrait la taille de la page en nombre de caractères pour constituer un tableau de 200 observations (pays) et 14 variables (langues). Par exemple la page pays de la France fait une taille de 129000 caractères dans l'édition en français, 126000 en anglais, 7000 en bengali, 800 en ourdou, etc.

Ce tableau est intéressant à plusieurs titres dans un cours d'introduction à la statistique :

- Utiliser des données qui sont des traces d'une activité numérique. Les données de ce type prennent une importance croissante, en particulier en géographie et en géomatique (Mericskay et al., 2018) et il est intéressant de s'y frotter dès la licence.

- Réfléchir au sens de la mesure : le nombre de caractère est-il une mesure correcte de la taille de la page, i.e. un caractère ourdou (alphabet perso-arabe) est-il comparable à un sinogramme chinois ou à une lettre de l'alphabet latin? 
- Construire des indicateurs dérivés : comment comparer des valeurs quand les ordres de grandeurs et les dispersions sont incomparables (la taille moyenne d'une page pays est de 61000 caractères en anglais et de 4600 caractères en ourdou) ?

- Donner du sens à une analyse de la forme des distributions (illustration 2): la distribution de l'anglais est la moins asymétrique (skewed), les autres langues étant très asymétriques. La symétrie de la distribution peut être utilisée comme marque de la complétude et de l'universalité de l'édition, au contraire la forte asymétrie dénote un focus sur un petit nombre de pays et une myopie sur le reste du monde.

Passer d'une mesure relativement pauvre à un raisonnement géographique (illustration 3) : peut-on mettre à jour une loi de proximité similaire à la loi du mort kilométrique dans l'analyse géomédiatique (Beauguitte et al., 2016) ?

Illustration 2 - Distribution de la taille des pages pays en Anglais et en Espagnol

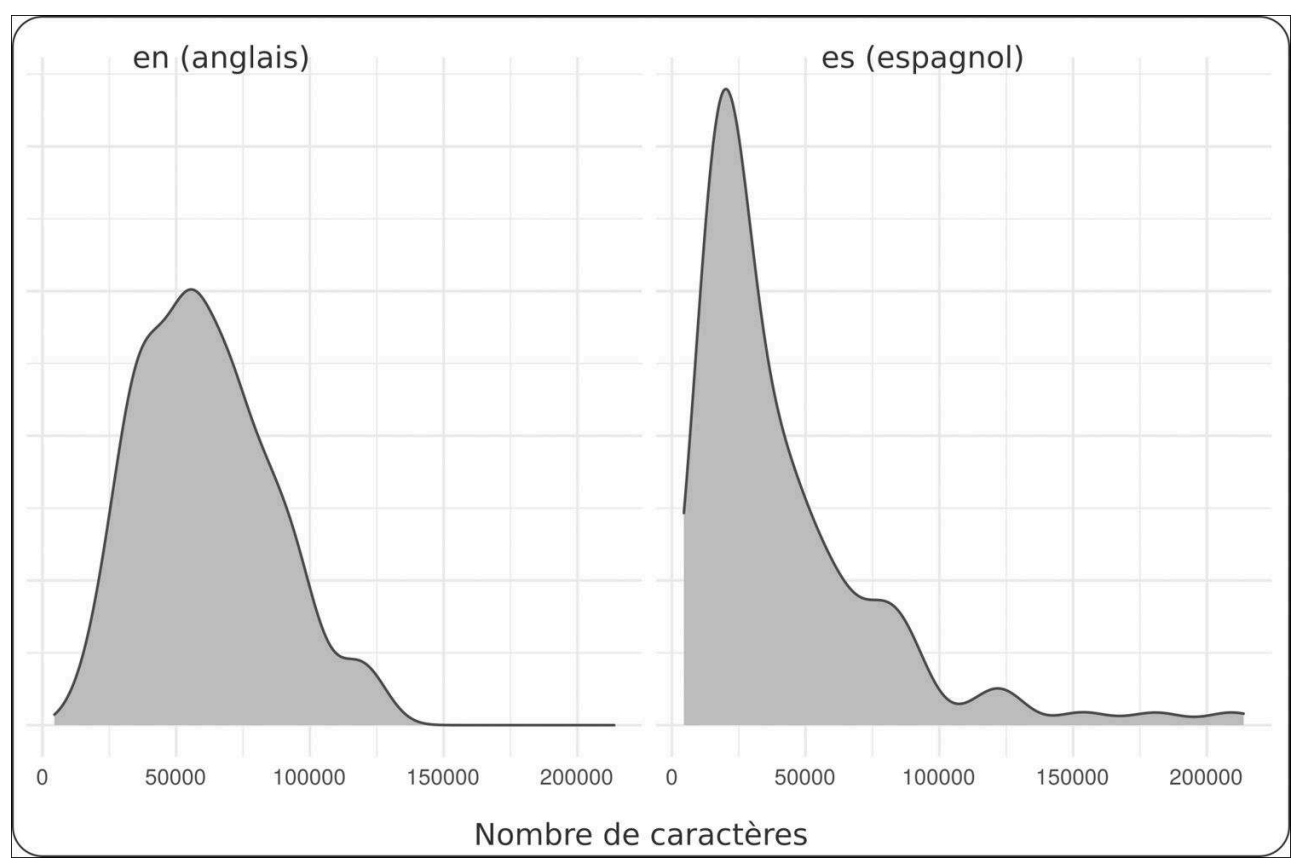

Un exemple d'analyse parmi d'autres (illustration 3b) : comparer la taille des pages pays dans l'édition anglaise et espagnole. Toute l'Espagne ainsi que toute l'Amérique hispanophone sont sur-éditées en espagnol. Deux pays montrent le statut particulier d'une langue officielle, véhiculaire, vis-à-vis des langues pratiquées, souvent en situation de domination symbolique. La Guinée équatoriale, pays dans lequel l'espagnol est langue officielle, mais parlée en général comme seconde langue, est sur-éditée en espagnol. De même, Belize, pays dans lequel l'espagnol est la principale langue parlée, mais où l'anglais est la langue officielle, est sur-édité en anglais. 
Illustration 3 - Taille des pages pays dans Wikipédia
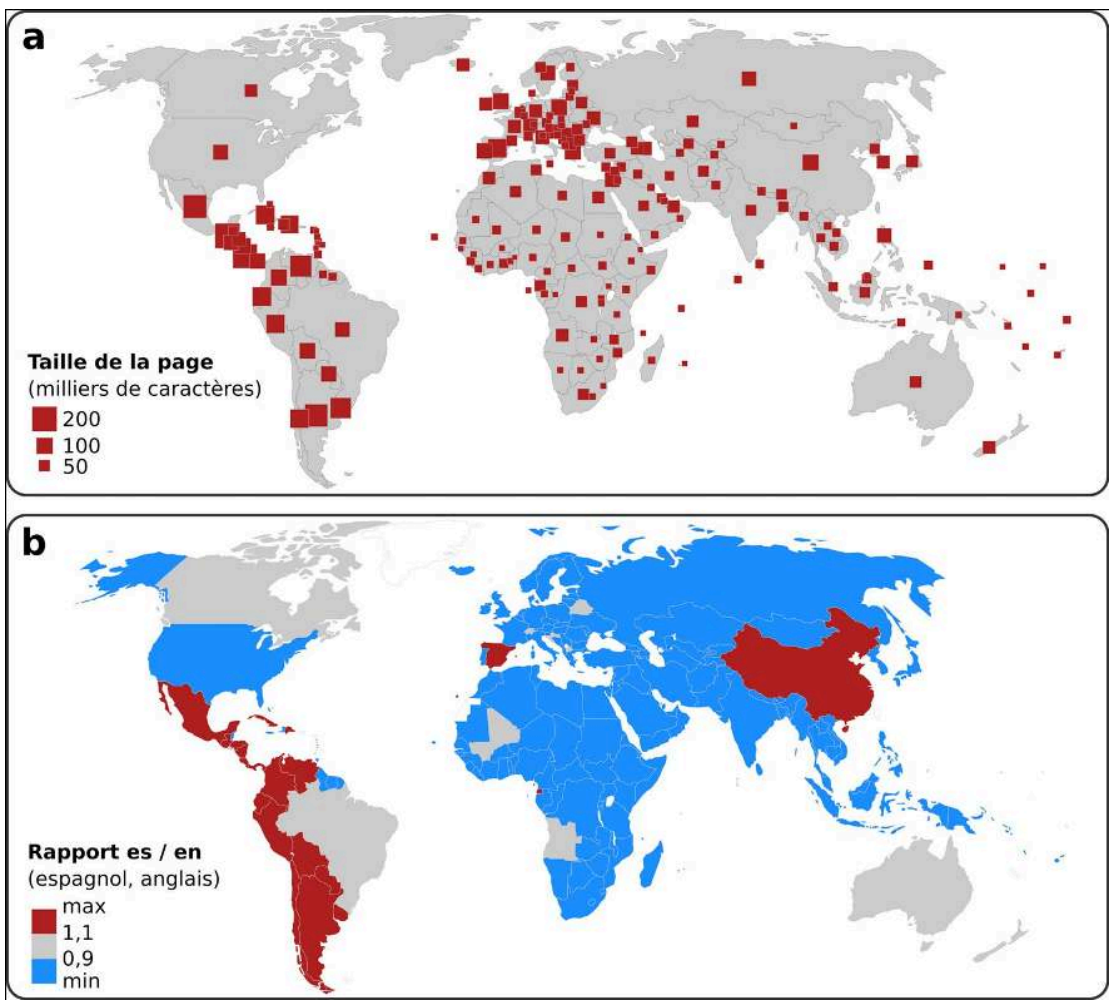

a) Valeur absolue dans l'édition espagnole. b) Rapport entre la taille dans les éditions espagnole et anglaise.

\section{Wikipédia comme dispositif d'écriture collaborative}

Dans un grand nombre de modules de géographie on demande aux étudiants un travail, souvent évalué, qui consiste à choisir de façon plus ou moins contrainte un sujet d'étude, à faire un état de l'art puis à rédiger un document synthétique sur le sujet. Le public visé est le seul enseignant évaluateur, et la motivation pour écrire un tel document repose essentiellement sur l'évaluation. Ce travail n'est en général plus relu au-delà de l'évaluation, ni par l'étudiant ni par l'enseignant. Pourtant le travail de documentation et de synthèse est le cœur de l'activité de Wikipédia, et faire ce travail non pour l'enseignant mais pour l'encyclopédie peut modifier profondément la nature de ce travail. C'est pour tester cette hypothèse que j'ai conçu en 2017 un nouveau programme pour un module d'études urbaines dans le cadre du cursus de L3. J'en décris ici les principes et reviens sur les effets de ce changement. Les contenus textuels et documents graphiques donnés comme exemple dans cette section ont été produits par les étudiants de ce module.

Le module compte en général 18 étudiants, il se fait sur 11 séances de $3 \mathrm{~h}$ dans une salle informatisée. La première séance sert de mise en route: présentation succincte de l'encyclopédie, jusqu'à présent toujours nécessaire puisque aucun étudiant de ces groupes n'était contributeur auparavant et proposition des sept thèmes qui constituaient le programme du cours avant le changement de démarche :1) définition de l'urbain, 2) étalement urbain, 3) mobilité et transport, 4) ségrégation et gentrification, 5) commerces, équipements, services, 6) logement, 7) réseaux 
techniques. À partir des préférences déclarées par les étudiants six groupes de trois étudiants sont constitués, correspondant à six des thèmes proposés.

Une fois les groupes constitués, les instructions sont les suivantes, à noter que, hormis le premier point, toutes ces actions ne sont pas faites une fois pour toutes, mais sont redéfinissables tout au long du projet :

1 - Faire un état des lieux sur le thème choisi des pages existantes dans Wikipédia.

2 - Dresser un diagramme programmatique avec les pages à modifier et, si besoin, à créer $^{23}$ et structurer ce programme en distinguant, a minima, notions principales, notions reliées et études de cas (illustration 4).

3 - Organiser les tâches au sein du groupe en fonction des envies et des besoins. Toutes les organisations sont possibles entre polyvalence (tout le monde agit de façon semblable) et spécialisation (chacun assume une fonction spécifique dans le projet d'édition).

4 - Tenir un journal de bord du projet éditorial, journal qui retrace à la fois les idées, les réalisations et les contacts avec les autres Wikipédiens.

Finalement, le rendu est un dossier qui retrace le parcours: il s'agit pour chaque groupe d'extraire la liste des contributions, de structurer les contributions par type et par importance, de commenter les contributions les plus significatives, d'adopter une démarche réflexive sur le travail accompli et sur les rapports avec les autres contributeurs. Ce dossier n'est pas une liste de contributions mais bien une synthèse structurée du projet éditorial.

Du côté des étudiants, passer d'une démarche très répandue (commentaires de documents et dossiers thématiques) à une démarche wikipédienne apporte plusieurs apprentissages relativement nouveaux. Les étudiants réfléchissent à l'organisation collective du travail, sachant que je ne leur donne pas de consigne particulière à ce propos hormis celle de s'organiser. Dans certains groupes j'ai pu observer une forte spécialisation avec des rôles très circonscrits, certains se spécialisant en bibliographie, d'autres en structuration, d'autres en définition, d'autres en production d'images graphiques, cartographiques ou photographiques. La spécialisation est peut-être induite par le fait qu'elle existe déjà dans Wikipédia avec une grande variété de rôles étiquetés (WikiGraphiste, WikiPhotographe, WikiTarentule, WikiFée, etc.). J'ai pu observer aussi des groupes avec une grande polyvalence, sans rôle prédéfini, mais avec une coordination pour avancer sur le projet. Les deux types d'organisation fonctionnent bien.

24 Ils se heurtent à la question des droits attachés aux productions intellectuelles, ce qui amène à parler de licences, et de plagiat. Ces questions émergent bien sûr au moment de verser un document graphique dans Wikimedia Commons. Cette question se pose parfois lors de la réutilisation de matériaux produits par d'autres (illustration 7).

25 Ce travail se révèle être une excellente valorisation des enseignements de statistique et de cartographie. Lors de la première expérience menée, j'ai été surpris de constater que tous les étudiants étaient intéressés par la production de graphiques ou de cartographie statistique pour nourrir les articles (illustrations 5 et 6). Cet intérêt s'est répété chaque année, et il touchait même ceux qui considéraient les modules étiquetés méthodes et outils (statistique, cartographie, SIG) comme une corvée. transmission de l'information. À la fin du semestre un étudiant a dit «si j'avais su que 
c'était des gens comme nous qui écrivaient les articles de Wikipédia, j'aurais fait plus attention en les lisant ». La phrase fait un peu penser à celle de Groucho Marx : « I don't want to belong to any club that would accept me as a member ${ }^{27}$. Mais il ne faut surtout pas confondre cette conclusion avec les jugements préconçus de certains intellectuels français comme Pierre Assouline (n'importe qui écrit n'importe quoi). Cette conclusion est peut-être le plus grand succès de la démarche : elle montre que l'étudiant ayant participé à la production du contenu est capable de se faire une idée de sa valeur et de ses limites. Et d'ailleurs les chercheurs feraient bien d'appliquer cette sentence : sachant que les articles scientifiques sont écrits par des gens comme nous, nous devrions faire plus attention en les lisant.

Illustration 4 - Diagramme du projet sur le thème de la ségrégation (Luc-Bastien, Dabestani, Ben Raïes, Thibaud, 2017)

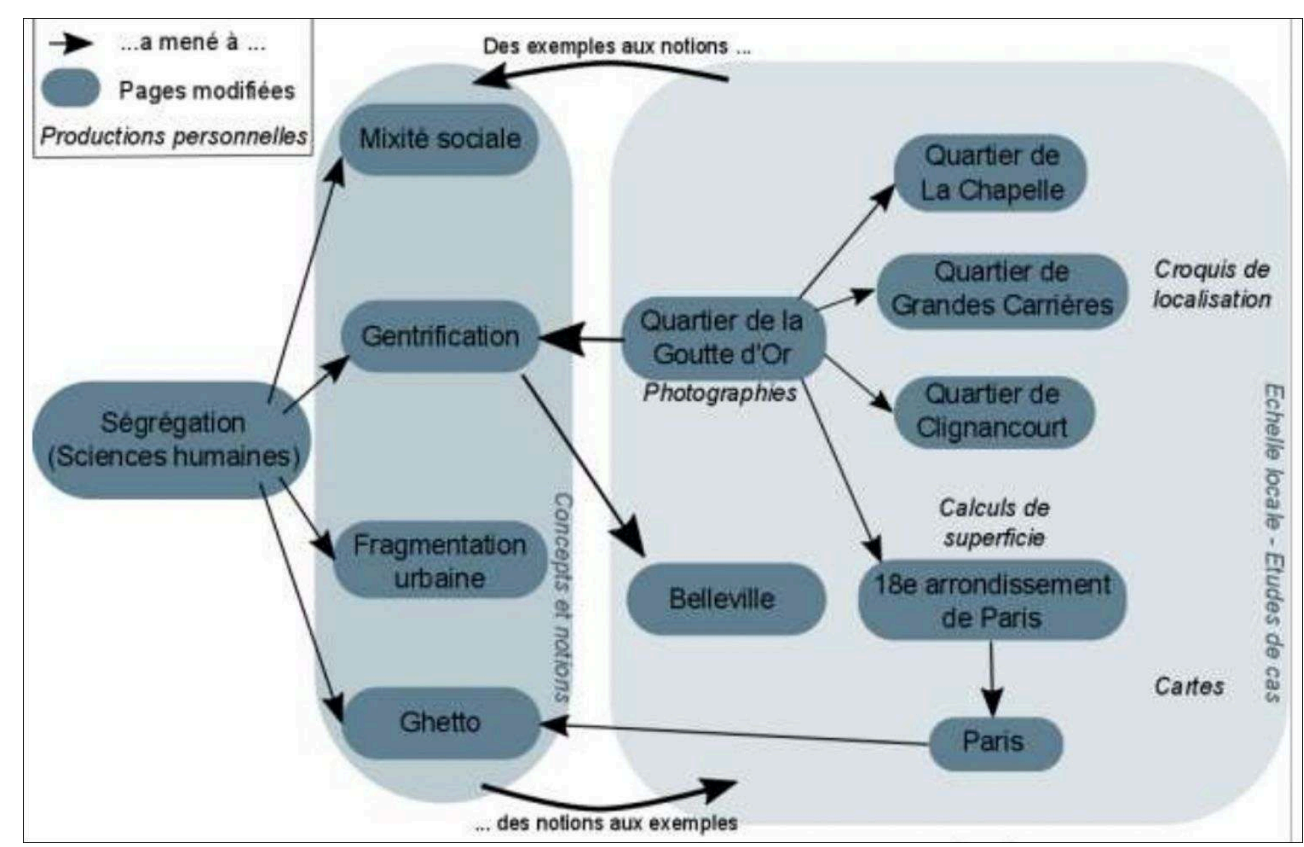


Illustration 5 - Évolution de la part des cadres et des ouvriers dans Paris (utilisateur Daryaana)

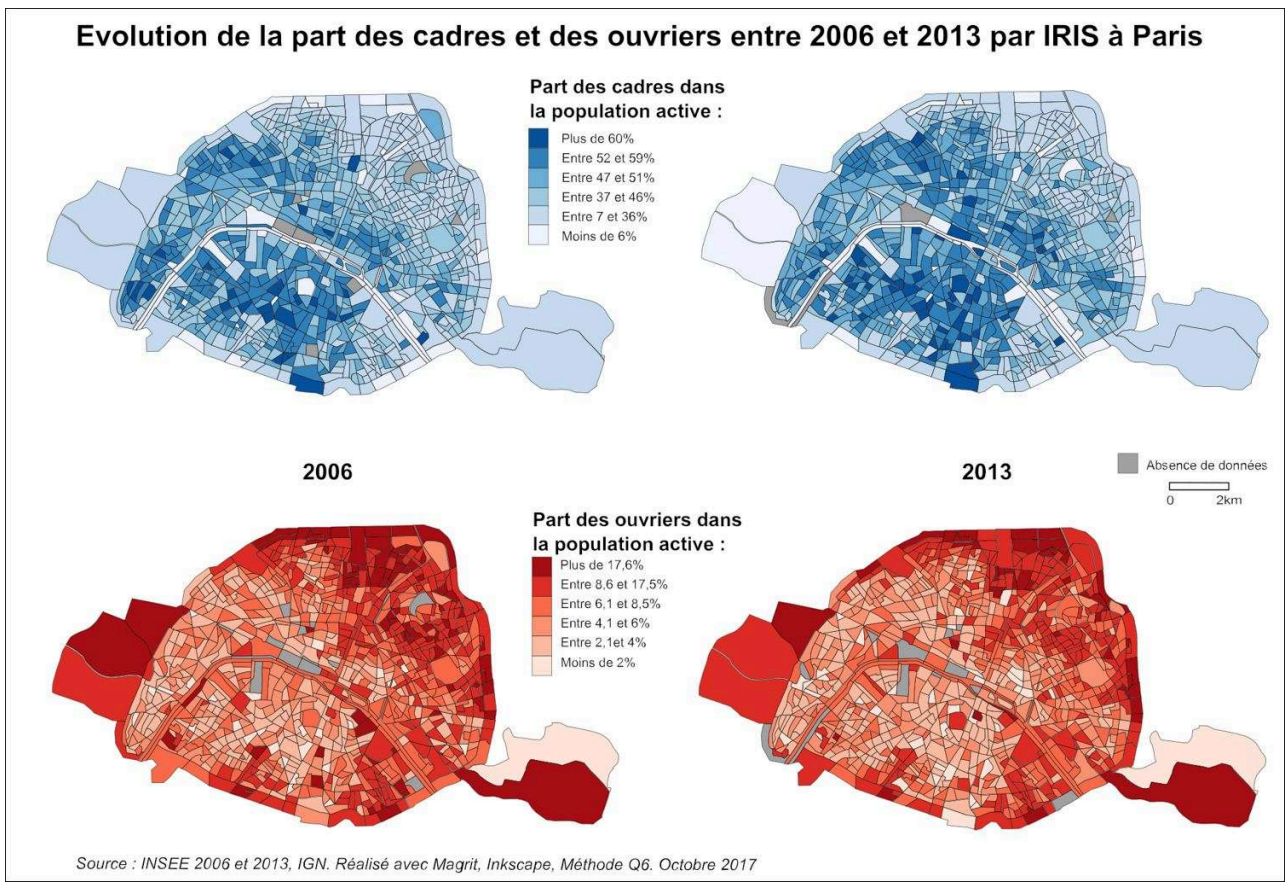

Illustration 6 - Évolution du mal-logement en France (utilisateur Tilen Le Gall)

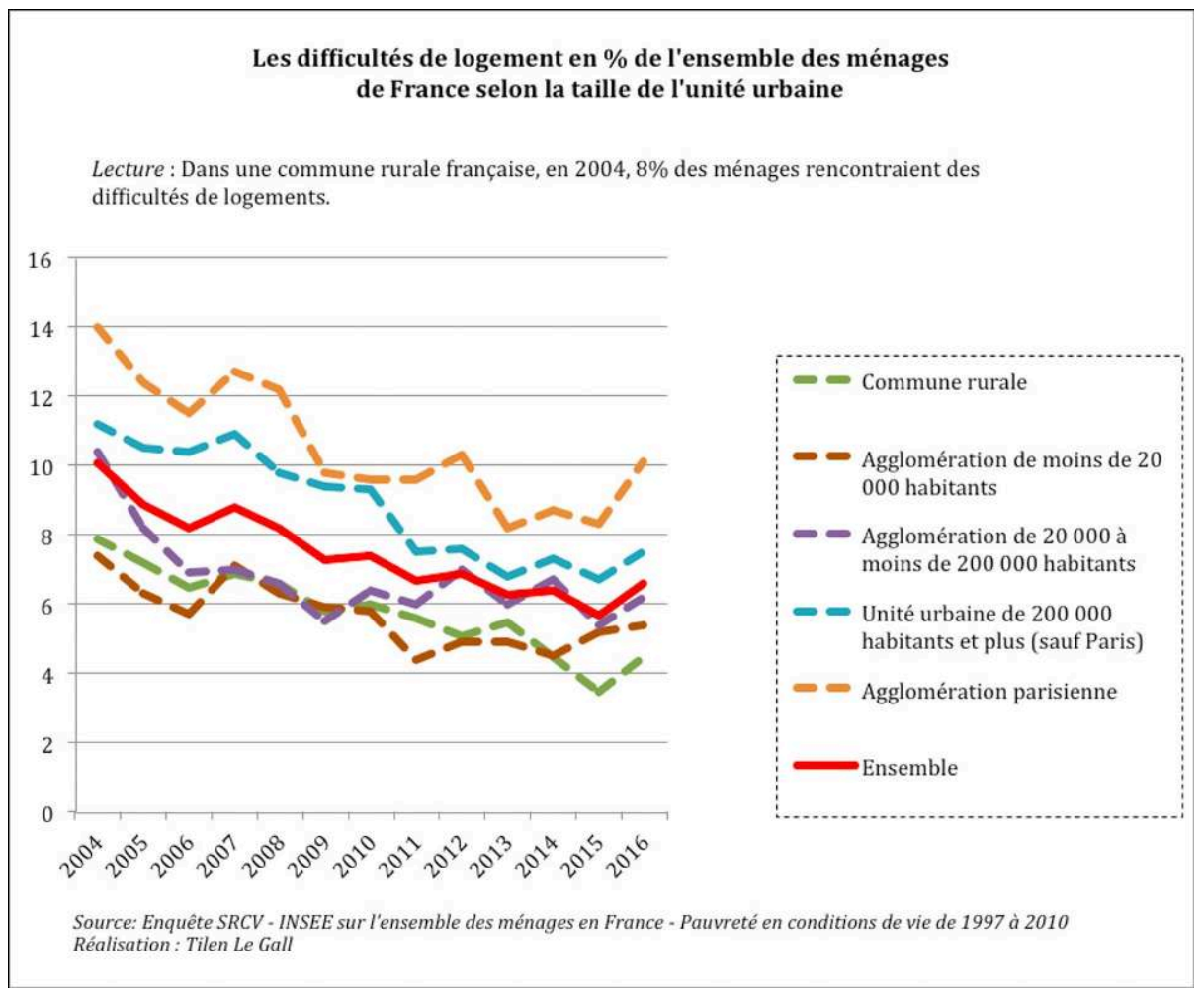


Illustration 7 - Photographie sur l'évolution quartier de la Goutte-d'Or (utilisateur Dhehz)

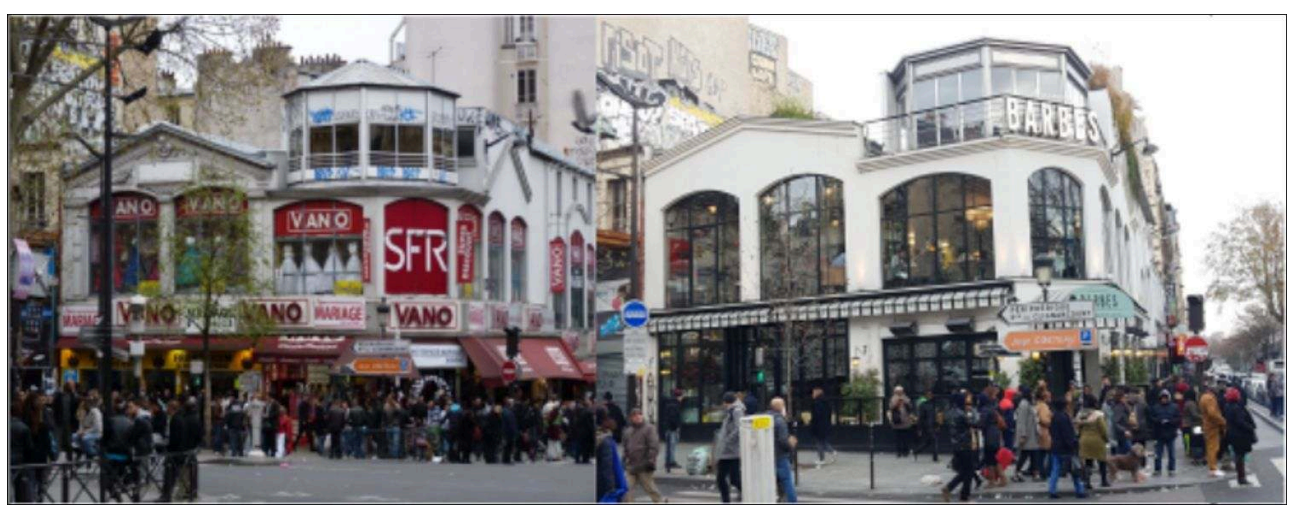

Du côté de l'enseignant, le travail consiste essentiellement à accompagner les étudiants dans le travail. La richesse mais aussi la difficulté de cet accompagnement consiste à s'adapter aux besoins de chaque étudiant et de chaque groupe. Dans un module classique l'enseignant s'adapte en partie, mais comme il est dans une position de transmission il est contraint de soutenir un certain flux d'information pour faire passer son programme. Dans une position d'accompagnement, le rythme est propre à chaque groupe, certains groupes feront plus, iront plus loin, mais ça n'a pas d'impact sur le rythme des autres. Les principales lignes d'accompagnement sont les suivantes:

- conseiller sur l'organisation des tâches ;

- guider dans la revue des contenus existants sur Wikipédia ;

- guider dans l'état de l'art bibliographique ;

- aider dans la production des documents, qu'il s'agisse de documents graphiques, cartographiques ou photographiques ;

- aider dans la structuration, la modification et la rédaction des textes.

Dans un module de ce type, il est ensuite facile de faire le suivi des contributions de chaque étudiant, chaque contributeur identifié ayant une page dédiée qui recense toutes ses contributions ${ }^{28}$. Pour pousser plus loin l'organisation d'un projet pédagogique avec Wikipédia il existe des outils dédiés pour afficher des objectifs, centraliser les contributions, etc. $^{29}$

\section{Wikipédia comme entrée dans le web sémantique}

Wikipédia peut être utilisée comme source d'information dans un mode de navigation (section 1), et c'est l'usage de la majorité. Wikipédia peut être utilisée comme dispositif d'écriture, dans lequel l'utilisateur devient contributeur (section 2), et cet usage mérite d'être développé dans un grand nombre de modules des cursus de licence et de master en géographie. Finalement, dans le cadre de certains masters où l'informatique occupe une place importante, Wikipédia peut être utilisée comme porte d'entrée vers le web sémantique. C'est cet usage qui est décrit ici à travers l'exemple d'un stage effectué en 2018 par Juliette Delannoy, étudiante du master Carthagéo ${ }^{30}$, au sein de l'UMR Géographie-cités, stage encadré par Thomas Louail et moi-même.

Le stage s'inscrivait dans le cadre d'un groupe de chercheurs, le groupe EIGHTIES, dont l'un des objectifs est de s'interroger sur les apports et les limites des nouvelles données pour la recherche en géographie, données issues du web, issues des capteurs ou encore des dispositifs GPS embarqués. L'objectif du stage était de produire un outil 
d'extraction d'information géographique utilisant les principaux outils du web sémantique. Cet outil visait à moissonner les sources de Volunteered Geographical Information (VGI), plus précisément d'explorer les possibilités offertes par la base DBPedia. Les objectifs d'apprentissage était introduire les principaux concepts du web sémantique, acquérir les bases du langage SPARQL, apprendre à concevoir et à développer une interface simple d'extraction de l'information.

Le web sémantique s'appuie sur la définition d'ontologies. Les ontologies sont des objets informationnels qui décrivent la nature et la structure des choses, et qui les organisent en catégories et en relations (Guarin et al., 2009). Ces catégories et ces relations sont encapsulées dans des bases de données de différentes façons, la spécification principale étant la spécification RDF (Ressource Description Framework) définie par le World Wide Web Consortium (W3C). Le RDF organise l'information en triplets sujet-prédicat-objet, chacun des éléments du triplet étant identifié de façon non équivoque par un URI (Uniform Ressource Identifier). C'est ce qui rend possible une sémantisation $d u$ web et des recherches que l'on peut formuler. Ce caractère sémantique est aujourd'hui intégré dans des outils communs, comme par exemple le moteur de recherche Google. Si on recherche Emil Zátopek, le moteur ne renvoie pas seulement des résultats associés à la chaîne de caractère Emil Zátopek, il renvoie aussi une infobox qui montre qu'il s'agit d'une chose de type "personne", et au sein de la catégorie "personne » d'une chose de type " athlète " puis les prédicats associés à ces catégories (date de naissance, lieu de naissance, médailles, etc.).

Cette information structurée peut être interrogée avec des requêtes sémantiques dont la richesse et la complexité dépendent à la fois de la richesse de la source d'information et de la maitrise du langage de requête. L'outil construit dans le cadre du stage, Let's Query DBPedia ${ }^{31}$, permet de lancer des requêtes simples sur DBPedia. Cet outil donne un aperçu du potentiel de cette base mais il est limité principalement sur deux aspects. D'abord il ne contient qu'une partie seulement des ontologies définies dans DBPédia ${ }^{32}$. Ensuite, comme il s'agit d'une interface graphique, il ne donne pas toute la marge de manœuvre du langage de requête lui-même (SPARQL).

Illustration 8 - Interface d'interrogation de DBPedia

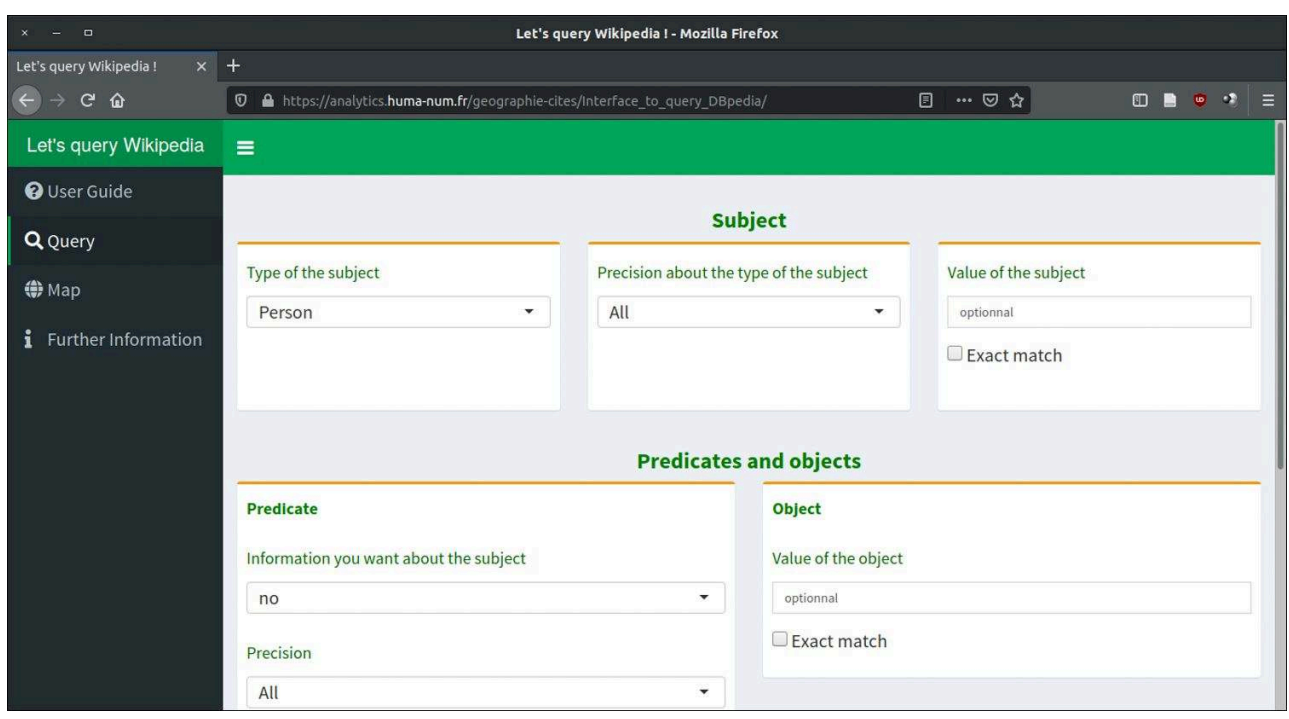


Ainsi, la requête la plus compliquée actuellement possible sur cette interface est de chercher un sujet, dont on peut préciser le type, et que l'on peut associer à deux prédicats. Par exemple : les personnes (sujet) de type politicien (précision sur le sujet) dont le lieu de naissance (prédicat 1) est en Afrique (objet du prédicat 1) et dont le lieu de mort (prédicat 2) est en France (objet du prédicat 2). Ou encore les personnes (sujet) de type artiste (précision sur le sujet) dont le lieu de naissance (prédicat 1) est Paris (objet du prédicat 1) et la date de naissance (prédicat 2) se situe entre 1900 et 1950 (objet du prédicat 2). Mais DBPedia contient un grand nombre d'ontologies qui seraient potentiellement interrogeables, par exemple il est possible d'extraire toutes les batailles impliquant le Canada ${ }^{33}$, ou toutes les batailles de la seconde guerre des Boers ${ }^{34}$. De plus, tous les emboîtements de triplets sujet-prédicat-objet sont théoriquement possibles : par exemple les personnes dont un parent est un artiste né à Paris entre 1900 et 1950. Dans cet exercice DBPedia a joué le rôle de terrain d'essai pour que l'étudiante s'approprie les principes du web sémantique. Pour le laboratoire, le stage a montré le potentiel d'une telle source d'information pour l'extraction d'information géographique.

\section{Conclusion}

Parmi les trois points qui ont été abordés, le second est peut-être le plus prometteur. Un grand nombre de modules des cursus de licence et de master de géographie pourraient comporter un projet éditorial sur Wikipédia. L'exemple qui a été décrit est, somme toute, assez proche des travaux déjà demandés dans les cours thématiques, de l'aménagement à l'hydrologie, qui consistent à rédiger des dossiers sur un sujet particulier au sein du domaine abordé dans le cours. D'autres exercices plus spécifiques peuvent être envisagés : en se focalisant sur l'écriture, obtenir le label qualité. En se focalisant sur les conflits, travailler sur des pages conflictuelles. Certaines pages font l'objet de dispositifs de médiation et de négociation ad hoc, par exemple les pages liées au conflit israélo-palestinien sont liées à un "salon de coopération $»^{35}$. Il serait sans doute intéressant de faire travailler les étudiants sur ce type de pages, en particulier dans des modules de géographie politique.

Du point de vue de mes pratiques d'enseignement, il y a un avant et un après l'expérience de projet éditorial mené en L3. C'est le changement d'attitude des étudiants qui marque le succès sans équivoque de cette approche. L'attitude des étudiants devant le travail est très différente de celle que j'ai pu constater dans des modules plus classiques du fait qu'ils peuvent s'approprier leur sujet et leurs pages, qu'ils peuvent faire voir leurs productions au monde plutôt qu'à leur seul enseignant. J'ai constaté un intérêt et une motivation bien supérieurs aux années précédentes. J'ai même parfois décelé une fierté devant certains accomplissements, sentiment que je n'ai jamais observé dans d'autres modules de licence. Fierté devant l'accomplissement, et non fierté devant la note. 


\section{BIBLIOGRAPHIE}

Assouline P., 2007. Wikipédia, l'erreur à haut débit. L'Histoire, no 318, p. 98.

Beauguitte L., Grasland C., Severo M., 2016. Espaces géographiques et représentations médiatiques. L'Espace Geographique, tome 45, no 1, p. 1-4.

Beauguitte L., Severo M., Pecout H., 2016. Une analyse géomédiatique de l'actualité internationale : hiérarchies et effets de voisinage. L'Espace Geographique, vol. 45, no 2, p. 124-141.

Broudoux E., 2015. Wikipédia, objet de recherches : entre observations, expérimentations et coconstructions. In Barbe L., Merzeau L., Schafer V., Wikipédia, objet scientifique non identifié. Nanterre, Presses universitaires de Paris Nanterre, p. 55-73.

Cardon D., Levrel J., 2009. La vigilance participative. Une interprétation de la gouvernance de Wikipédia. Reseaux , vol. 154, nº 2, p. 51-89.

Cummings R., 2019. The first twenty years of teaching with Wikipedia: from faculty enemy to faculty enabler. In Reagle J., Koerner J., Wikipedia @20. MIT Press.

Doutreix M.N., 2020. Wikipédia et l'actualité. Qualité de l'information et normes collaboratives d'un média en ligne. Paris, Presses Sorbonne Nouvelle.

Guarino N., Oberle D., Staab S., 2009. What is an ontology? In Staab S., Studer R., Handbook on ontologies. Berlin, Springer-Verlag, p. 1-17.

Haslam T.J., 2017. Wikipedia and the humanities in higher education: past time to renegotiate the relationship. International Journal of Information and Education Technology vol. 7, nº 4, p. 246-51.

Keegan B., Gergle K., Contractor N., 2013. Hot off the wiki : structures and dynamics of Wikipedia's coverage of breaking news events. American Behavioral Scientist, vol. 57, nํㅗ, p. 595-622.

Knight C., Pryke S., 2012. Wikipedia and the university, a case study. Teaching in Higher Education, vol. $17, \mathrm{n}^{\circ}$ 6, p. 649-659.

Levrel J., 2006. Wikipedia, un dispositif médiatique de publics participants. Reseaux, vol. 138, ํㅡ 4, p. $185-218$.

Medelyan O., Milne D., Legg C., Witten I.H., 2009. Mining meaning from Wikipedia. International Journal of Human-Computer Studies, vol. 67, nº 9, p. 716-754.

Mehdi M., Okoli C., Mesgari M., Nielsen F.A., Lanamäki A., 2017. Excavating the mother lode of human-generated text : a systematic review of research that uses the Wikipedia corpus. Information Processing \& Management, vol. 53, nํ 2, p. 505-529.

Mericskay B., Noucher M., Roche S., 2018. Usages des traces numériques en géographie : potentiels heuristiques et enjeux de recherche. L’Information Geographique, vol. 82, no 2, p. 39-61. Overell S., Rüger S., 2008. Using co-occurrence models for placename disambiguation. International Journal of Geographical Information Science, vol. 22, ํㅡ 3, p. 265-287.

Pentzold C., Weltevrede E., Mauri M., Laniado D., Kaltenbrunner A., Borra E., 2017. Digging Wikipedia : the online encyclopedia as a digital cultural heritage gateway and site. Journal on Computing and Cultural Heritage, vol. 10, nํ1, p. 1-19. 
Sahut G., Jeunier B., Mothe J., Tricot A., 2015. Qu'apprennent les jeunes usagers à propos de Wikipédia ? In Barbe L., Merzeau L., Schafer V. Wikipédia, objet scientifique non identifié. Nanterre, Presses universitaires de Paris Nanterre, p. 149-161.

Weltevrede E., Borra E., 2016. Platform affordances and data practices: the value of dispute on Wikipedia. Big Data \& Society, vol. 3, nº 1, p. 1-16.

\section{NOTES}

1. Source : Wikimedia Statistics, URL: https ://stats.wikimedia.org

2. URL: http://contropedia.net

3. URL: https://outreach.wikimedia.org/wiki/Wikipedian_in_Residence

4. URL: https://outreach.wikimedia.org/wiki/Education

5. URL: https://fr.wikipedia.org/wiki/Wikip\%C3\%A9dia:Projets_p\%C3\%A9dagogiques

6. Voir par exemple le projet mené par Lionel Barbe dans le cadre du master Communication Rédactionnelle Dédiée au Multimédia de l'université de Nanterre, URL: https:// fr.wikiversity.org/wiki/Projet:Valoriser_ses_connaissances_avec_Wikip\%C3\%A9dia/

Communication_R\%C3\%A9dactionnelle_D\%C3\%A9di\%C3\%A9e_au_Multim\%C3\%A9dia_Universit\%C3\%A9_Paris_2013

7. «Wikipédia peut et doit jouer un rôle essentiel dans l'enseignement supérieur des sciences humaines, notamment en préparant les étudiants à mieux écrire et à effectuer des recherches scientifiques. ».

8. Les fichiers média qui ne sont pas du domaine public sont en général protégés par une licence Creative Commons BY-SA qui indique l'obligation d'attribuer l'œuvre à son auteur (BY) et l'obligation de partager les œuvres dérivées selon les mêmes conditions (SA).

9. URL : https://commons.wikimedia.org/wiki/File:Delhi,_Great_Capital_of_India_(1909).webm

10. URL : https://commons.wikimedia.org/wiki/File:Bundesarchiv_B_145_BildF010324-0002,_Flughafen_K\%C3\%B6ln-Bonn,_Adenauer,_de_Gaulle-cropped.jpg

11. URL : https://commons.wikimedia.org/wiki/

File:President_Mitterand_bij_slotzitting_Europa_Congres_Mitterand,_kop,_Bestanddeelnr_934-2444_(portrait_crop).jpg

12. URL : https://commons.wikimedia.org/wiki/File:Emmanuel_Macron_in_2019.jpg

13. «Ce fichier est une œuvre d'un employé du Bureau exécutif du Président des États-Unis, prise ou réalisée dans le cadre des fonctions officielles de cette personne. En tant qu'œuvre du gouvernement fédéral américain, il est dans le domaine public. »

14. URL : https://en.wikipedia.org/wiki/List_of_lists_of_lists

15. URL : https://en.wikipedia.org/wiki/List_of_riots

16. URL : https://en.wikipedia.org/wiki/List_of_epidemics

17. URL : https://en.wikipedia.org/wiki/List_of_international_border_rivers

18. URL : https://fr.wikipedia.org/wiki/Liste_de_langues_par_date_d\%27extinction

19. URL: https://github.com/search?q=wikipedia+crawler

20. URL: https://wiki.digitalmethods.net/Dmi/ToolDatabase

21. URL: http://tools.medialab.sciences-po.fr

22. Il s'agit des langues suivantes : arabe, aengali, allemand, anglais, espagnol, français, hindi, indonésien, italien, japonais, portugais, russe, ourdou, chinois.

23. La plus grande partie du travail a été effectuée sur des pages déjà existantes, avec quelques exceptions notables comme la création et le développement de la page "Déclin urbain". URL: https://fr.wikipedia.org/wiki/D\%C3\%A9clin_urbain.

24. URL: https://commons.wikimedia.org/wiki/File:Evolution_paysag\%C3\%A8re_Barb\%C3\%A8sRochechouart.png 
25.

URL:

https://commons.wikimedia.org/wiki/

File:Carte_de_1\%27\%C3\%A9volution_de_la_part_des_ouvriers_et_des_cadres_par_IRIS_\%C3\%A0_Paris_en_2006_et_2013.svg

26.

URL:

https://commons.wikimedia.org/wiki/

File:Les_difficult\%C3\%A9s_de_logement_en_\%25_de_l\%27ensemble_des_m\%C3\%A9nages_de_France,_selon_la_taille_de_l\%27unit\%C3\%

27. " Je ne veux pas appartenir à un club qui m'accepterait comme membre. »

28. Voici à titre d'exemple la page utilisateur de Fbarfr, étudiant de la première édition de ce cours : https://fr.wikipedia.org/wiki/Sp\%C3\%A9cial:Contributions/Fbarfr. On peut y suivre chaque publication faite sur l'encyclopédie. Il est également possible d'utiliser l'outil Wikiscan pour afficher des statistiques agrégées de chaque contributeur. Toujours pour ce contributeur, l'outil https://fr.wikiscan.org/utilisateurs/Fbarfr montre 23 publications au total entre septembre et décembre 2017 , et un total de $17 \mathrm{k}$ octets de modifications.

29. Voir la page dédiée pour plus d'informations sur ces outils et pour des exemples de projets pédagogiques, URL: https://fr.wikipedia.org/wiki/ Wikip\%C3\%A9dia:Projets_p\%C3\%A9dagogiques/Projets

30. Il s'agit plus précisément d'un stage de 5 semaines, dit stage de développement, qui concerne uniquement les étudiants de l'École Nationale des Sciences Géographiques.

31. L'application est développée avec $\mathrm{R}$ et shiny et hébergée sur le serveur du TGIR Huma-Num à l'adresse suivante : https://analytics.huma-num.fr/geographie-cites/ Interface_to_query_DBpedia/.

32. URL: http://mappings.dbpedia.org/server/ontology/classes

33. URL: http://dbpedia.org/page/Category:Battles_involving_Canada

34. URL: http://dbpedia.org/page/Category:Battles_of_the_Second_Boer_War

35. URL: https://fr.wikipedia.org/wiki/Projet:Salon_de_coop\%C3\%A9ration_Isra\%C3\%ABlPalestine

\section{RÉSUMÉS}

Depuis sa naissance, Wikipédia a fait l'objet de nombreux travaux dans des disciplines variées : informatique, linguistique, géographie, science politique, etc. L'usage de Wikipédia comme objet de recherche n'a jamais posé problème, en revanche, l'usage de Wikipédia pour l'enseignement, en particulier pour l'enseignement universitaire, a toujours posé problème. Une majorité d'enseignants sont méfiants vis-à-vis de l'encyclopédie et recommandent explicitement aux étudiants de ne pas l'utiliser. Malgré ces recommandations, les élèves et les étudiants utilisent massivement Wikipédia pour la recherche d'information, tout en considérant, après leurs enseignants, qu'il s'agit d'une source illégitime. Ainsi, l'encyclopédie dispose d'un statut ambivalent, elle est à la fois utilisée et méprisée par les étudiants et les enseignants alors que très peu d'entre eux sont contributeurs. Wikipédia reste très peu intégrée aux enseignements universitaires alors qu'elle présente des avantages certains. Dans ce texte je propose un retour d'expérience issu de trois modules donnés dans l'UFR de géographie de l'Université Paris 1 Panthéon Sorbonne. À partir de ces expériences je mets en avant l'intérêt que représente l'usage de Wikipédia pour l'enseignement universitaire, en SHS et en géographie en particulier.

Wikipedia has always been a research object in several disciplinary fields: computer science, linguistics, geography, political science... Taking Wikipedia as a research object has never been a problem, but using Wikipedia for teaching, especially for university teaching, has always been a 
problem. A majority of teachers have a suspicious look at the encyclopedia and explicitly recommend students not to use it. Despite these recommendations, students massively use Wikipedia for information retrieval, while considering, after their teachers, that it is an illegitimate source of information. Thus, the encyclopedia has an ambivalent status, it is both used and despised by students and teachers while very few of them are contributors. Wikipedia remains an outsider for university teaching, even though it has many advantages. In this text I propose a feedback from three classes given in the Geography Department of the University of Paris 1 - Panthéon Sorbonne. Based on these experiences, I highlight the interests of using Wikipedia for geography teaching.

\section{INDEX}

Thèmes : Sur le Métier

Keywords : Wikipedia, digital humanities, teaching, collaborative writing

Mots-clés : Wikipédia, humanité numérique, enseignement, écriture collaborative

\section{AUTEUR}

\section{HADRIEN COMMENGES}

Hadrien Commenges, Hadrien.Commenges@univ-paris1.fr, est Maître de conférences à l'Université Paris 1 Panthéon-Sorbonne et membre de l'UMR 8504 Géographie-cités. Il a récemment publié :

- Delage M., Baudet-Michel S., Fol S., Buhnik S., Commenges H., et al., 2020. Retail decline in France's small and medium-sized cities over four decades. Evidences from a multi-level analysis. Cities, vol. 104.

- Raimbault J., Chasset P.-O., Cottineau C., Commenges H., et al., 2019. Empowering open science with reflexive and spatialised indicators., Environment and Planning $B$.

- Li Y., Commenges H., Bordignon F., et al., 2018. The Tianjin eco-city model in the academic literature on urban sustainability. Journal of Cleaner Production, vol. 213, p. 59-74. 